\title{
The Effect of Cell-Free Supernatants of Free-Living Amoeba against Some Staphylococcus Bacteria: First Findings from Turkey
}

\author{
Sevval Maral Ozcan ${ }^{1,2}$ (D), Zuhal Zeybek ${ }^{3}$ \\ IIstanbul University, Institute of Science, Istanbul, Turkey \\ ${ }^{2}$ Biruni University, Faculty of Pharmacy, Department of Pharmacy, Pharmaceutical Microbiology Division, Istanbul, Turkey \\ ${ }^{3}$ Istanbul University, Faculty of Science, Department of Biology, Fundamental and Industrial Microbiology Division, Istanbul, Turkey
}

ORCID IDs of the authors: S.M.O. 0000-0002-3336-5618; Z.Z. 0000-0002-9407-9519

Please cite this article as: Ozcan SM, Zeybek Z. The Effect of Cell-Free Supernatants of Free-Living Amoeba against Some Staphylococcus Bacteria: First Findings from Turkey. Eur J Biol 2021; 80(1): 29-34. DOI: 10.26650/EurJBiol.2021.927747

\section{ABSTRACT}

Objective: Free-living amoeba (FLA) are protozoa living in soil and in natural and man-made water systems. They attract much attention owing to the illnesses associated with them and to their relationships with bacteria. In this study, the effect of cell-free supernatant (CFS) obtained from FLA against Staphylococcus was investigated.

Materials and Methods: Environmental FLA strains (A1, A2, A3) were obtained from lake water and swimming pools in Istanbul. Acanthamoeba castellanii ATCC 50373 was used as the standard strain. Clinical Staphylococcus strains (S1, S2, S3) were obtained from a culture collection at Istanbul University, Faculty of Pharmacy, Department of Pharmaceutical Microbiology. As standard strains, MRSA ATCC 43300, S. epidermidis ATCC 12228, S. aureus ATCC 29213 were used. FLA-CFS were obtained by centrifuging and filtering of axenic cultures. Colony counting technique was used to investigate the inhibition activities of FLA-CFS against Staphylococcus bacteria.

Results: Against MRSA ATCC 43300 strain, CFSs of A. castellanii ATCCC 50373 and A1 showed an inhibition efficiency of $78.36 \%$ and $73.47 \%$, respectively. Against S1 strain, CFSs of A. castellanii ATCCC 50373 and A2 showed an inhibition of $65.64 \%$ and $15.14 \%$, respectively. Against S. aureus ATCC 29213 , only A1-CFS showed inhibitory effect (44\%). It was found that $A$. castellanii ATCC 50373 and A2-CFSs inhibited the S2 strain $26.20 \%$ and $9.24 \%$ respectively. Against S3 strain, A2-CFS was inhibitory at $33.33 \%$. No FLA-CFS could be inhibitory against S. epidermidis ATCC 12228.

Conclusion: It is necessary to devise new studies in which sample numbers are increased when using FLA strains in the inhibition of antibiotic-resistant bacteria.

Keywords: Free-living amoeba, Staphylococcus infections, Acanthamoeba, antibacterial effect, cell-free supernatant, antibiotic resistance

\section{INTRODUCTION}

Free-living amoeba (FLA) are eukaryotic microorganisms living in soil, air, sea water, fresh water and manmade water systems (pools, cooling towers, water pipes, etc.) (1-8). They have two stages in their lifecycle, namely trophozoite and cyst (9). Trophozoites are metabolically active, feeding, mobile forms which transform into cysts. The latter are inactive metabolically and exist in adverse environmental conditions (starvation, temperature changes, $\mathrm{pH}$ changes, etc.) (10). Pathogenic FLA for humans are Naegleria fowleri, Acanthamoeba spp., Balumuthia mandrillaris, and Sappinia spp $(5,11)$. Along with their pathogenicities, FLA also attracted scientists' attention due to their interactions with other microorganisms in the environments in which they live. In this collective living, they play important roles in the 
regulation of microorganism population and microbial ecology $(12,13):$ i) They use some bacteria, fungi, and algae as food $(10,14,15)$. ii) Some bacteria which can enter inside FLA find themselves isolated from adverse environmental conditions (antibiotics, disinfectants, etc.) and they find the opportunity to multiply (16-19). After this, they can cause lysis of FLA cells (20). iii) They can inhibit some bacteria which does not enter inside FLA cells (non-fagocyting). Very scarce studies in recent years show these abilities (21-24).

Recently, increasing antibiotic resistance has become an important problem in the treatment of bacterial infections. Every year, about 2.9 million infections owing to the presence of antibiotic-resistant bacteria occur and about 36 thousand of them end in death (25). From past to present, antibacterial substances have been isolated from organisms like bacteria, fungi, algae, insects, and plants (26-29).

As is the case all around the world, new antibacterial compounds are also sought after in our country because antibiotic-resistant bacteria cause important problems in the treatment of these infections. However, the number of studies showing the antibacterial effect of FLA is quite limited in the literature $(22,23)$. So, in this context, this research aims to look for the antibacterial effect of cell-free supernatant (CFS) of FLA strains isolated in Turkish waters against infection-agent Staphylococcus bacteria.

\section{MATERIALS AND METHODS}

\section{Test Microorganisms}

In this study, the antibacterial activity of CFS obtained from four FLA (Acanthamoeba castellanii ATCC 50373, A1, A2, A3) was investigated against six Staphylococcus strains [Methicil- lin-resistant Staphylococcus aureus ATCC 43300 (MRSA ATCC 43300), Staphylococcus aureus ATCC 29213 (S. aureus ATCC 29213), Staphylococcus epidermidis ATCC 12228 (S. epidermidis ATCC 12228), Methicillin-resistant Staphylococcus aureus S1 (S1), Methicillin-sensitive Staphylococcus aureus S2 (S2), Methicillin-resistant Staphylococcus epidermidis S3 (S3)] (Table 1). Among the FLA strains used in this study, two of them (A1 and A2) were isolated from swimming pools in a previous study of ours (8), one of them (A3) was isolated from lake water, and A. castellanii ATCC 50373 was used as standard strain. Clinical Staphylococcus strains (S1, S2, S3) used in our study were obtained from the culture collection at Istanbul University (I.U.) Faculty of Pharmacy, Department of Pharmaceutical Microbiology. MRSA ATCC 43300, S. aureus ATCC 29213 and S. epidermidis ATCC 12228 were used as the standard strains.

\section{Bacterial Cultures}

MRSA ATCC 43300, S. aureus ATCC 29213, S. epidermidis ATCC 12228, S1, S2 and S3 strains were cultured in Trypticase soy agar (TSA) at $37^{\circ} \mathrm{C}$ for 24 hours and used in the experiment.

\section{FLA Cultures}

FLA strains (A1, A2) previously isolated and kept in the freezing medium (FM) at $-86^{\circ} \mathrm{C}$ were first brought to room temperature. Petri dishes containing non-nutrient agar (NNA) spread on Escherichia coli (E. coli) pre-inactivated $\left(121^{\circ} \mathrm{C}, 15\right.$ minutes) were used for the resuscitation of the strains. All Petri dishes were incubated for 3 to 4 weeks at $30^{\circ} \mathrm{C}$ and they were examined under inverted microscope every day $(30,31)$. Dense FLA trophozoite-bearing areas in NNA were marked and cut with a sterile lancet, and patched on fresh NNA medium surface (with inactivated E. coli) upside-down $(2,32,33)$. All Petri dishes were incubated at $30^{\circ} \mathrm{C}$.

Table 1. Microorganisms used in antibacterial activity experiments.

\begin{tabular}{|c|c|c|}
\hline Microorganisms (Code) & & Source \\
\hline \multirow{4}{*}{ FLA Strains } & Acanthamoeba castellanii ATCC 50373 & Standard Strain \\
\hline & A1 & Swimming pool isolate \\
\hline & $\mathrm{A} 2$ & Swimming pool isolate \\
\hline & $\mathrm{A} 3$ & Lake water isolate \\
\hline \multirow{6}{*}{ Staphylococcus Strains } & aMRSA ATCC 43300 & Standard Strain \\
\hline & 'bS. aureus ATCC 29213 & Standard Strain \\
\hline & 'S. epidermidis ATCC 12228 & Standard Strain \\
\hline & dS1 & Clinical isolate \\
\hline & es2 & Clinical isolate \\
\hline & fS3 & Clinical isolate \\
\hline \multicolumn{3}{|c|}{$\begin{array}{l}\text { a: Methicillin-resistant Staphylococcus aureus ATCC 43300, b: Methicillin-sensitive Staphylococcus aureus ATCC 29213, } \\
\text { c: Methicillin-sensitive Staphylococcus epidermidis ATCC 12228, d: Methicillin-resistant Staphylococcus aureus, } \\
\text { e: Methicillin-sensitive Staphylococcus aureus, f: Methicillin-resistant Staphylococcus epidermidis }\end{array}$} \\
\hline
\end{tabular}


First, A3-coded amoeba strain was isolated from Uluabat Lake (Bursa, Turkey) under aseptic conditions, and the water sample in a glass bottle was concentrated by passing through a Sartorius filtering device having a membrane filter $(0.22 \mu \mathrm{m})$. Then, the filter paper was turned upside down and left on fresh NNA medium having inactivated $E$. coli and incubated at $30^{\circ} \mathrm{C}(4,34)$. Petri dishes were examined on an inverted microscope on a daily basis.

\section{FLA-Axenic Culture and FLA-CFS}

To obtain FLA-axenic cultures, Pepton Yeast Extract Glucose medium (PYG) containing Page's amoeba saline (PAS) solution (PYG-PAS) was used $(11,35)$. Antibiotics $(0.5 \mathrm{mg} / \mathrm{mL}$ penicillin and streptomycin) were added to PYG-PAS to avoid microbial contamination. Amoeba cells in the Petri dishes were collected with 2-3 mL of PAS solution and inoculated into T-25 tissue culture flask containing antibiotic-added PYG-PAS and incubated at $30^{\circ} \mathrm{C}(22,36)$. When the mono-layered axenic culture was observed, the PYG-PAS containing antibiotics in the tissue culture flask was discharged and washed with fresh PAS three times, in order to clean the culture from antibiotics (17). After the last washing, fresh PYG-PAS was placed into the flask and incubated for $48-72$ hours at $30^{\circ} \mathrm{C}$. The FLA-axenic culture thus obtained was subjected to Thoma slide and trypan blue for counting (cell/ $\mathrm{mL}$ ) and vitality determination $(37,38)$. Cell-free supernatant of FLA (FLA-CFS) was obtained after centrifuging the FLA-axenic cultures (1000 $\mathrm{g} \times 5 \mathrm{~min}$ ) followed by passing the supernatant through $0.22 \mu \mathrm{m}$ pore-diameter filters and were used in antibacterial experiments $(22,23)$.

\section{Antibacterial Activity Experiments}

Colony counting method was used to assess the antibacterial effect of FLA-CFSs against Staphylococcus bacterial strains (22, 23). Bacterial suspension $\left(10^{6} \mathrm{cfu} / \mathrm{mL}\right)$ in phosphate buffered saline (PBS) and FLA-CFS were mixed in a tube (1:10) and incubated for $18-24$ hours at $37^{\circ} \mathrm{C}$. After incubation, each mixture was diluted further with PBS 10 times to achieve a series of dilutions $\left(10^{-1}-10^{-6}\right)$ and spread into Petri dishes containing TSA. All petri dishes were incubated for 24 hours at $37^{\circ} \mathrm{C}$ and after the period, colonies were counted. In the experiments, PYG-PAS and PBS were used as control groups. All experiments were performed in triplicate.

\section{RESULTS}

\section{FLA-Axenic Culture and FLA- CFS}

Figure 1 shows the images obtained under inverted microscopic examination (x100) of FLA-axenic cultures.

Each FLA-axenic culture had the following living FLA trophozoite numbers: For A. castellanii ATCC 50373, A1, A2, A3, the values are $3 \times 10^{5}, 8 \times 10^{5}, 5 \times 10^{5}, 1 \times 10^{5}$ cell $/ \mathrm{mL}$, respectively. Trypan blue-dyed FLA-axenic cultures had the microscopic view (x100) of undyed/living FLA trophozoites in Figure 2.

\section{Antibacterial Activity}

The tested FLA-CFSs showed the highest inhibition effect on the MRSA ATCC 43300 strain among the Staphylococcus spe-

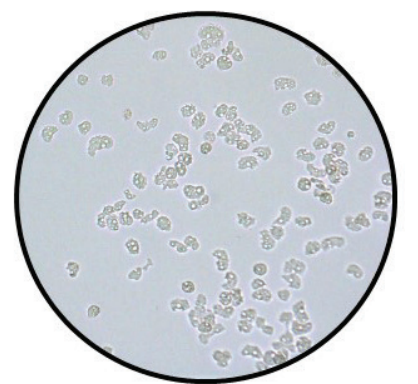

A

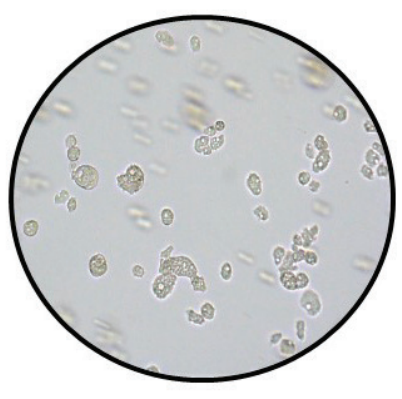

C
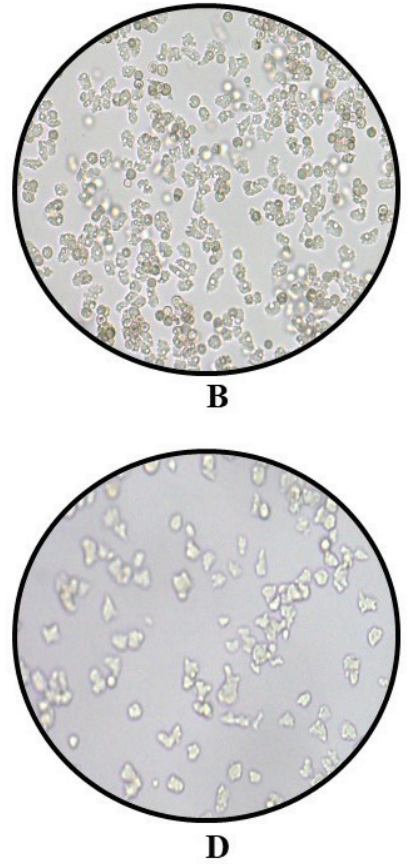

D
Figure 1. Microscopic images of axenic cultures obtained from each FLA (x100): A) Acanthamoeba castellanii ATCC 50373, B) A1, C) $A 2, D) A 3$.

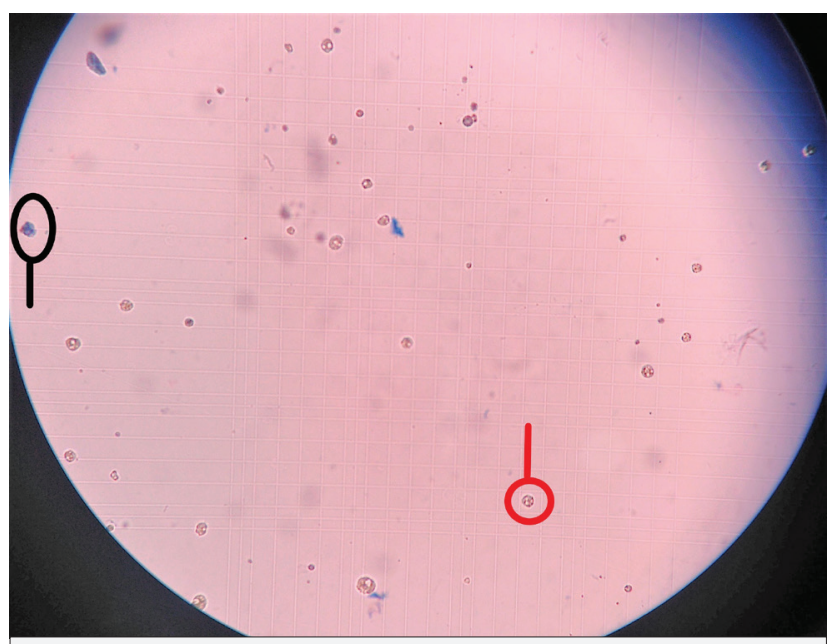

Figure 2. Light microscopic view (x100) of axenic culture of Acanthamoeba castellanii ATCC 50373 strain on the trypan bluedyed Thoma slide; live trophozoite (red labelled) and dead trophozoite (black labelled).

cies tested (Figure 3). A. castellanii ATCCC 50373 coded FLA ( $A$. castellanii ATCCC 50373-CFS) and A1 encoded FLA-CFS (A1-CFS) inhibited MRSA ATCC 43300 bacteria by $78.36 \%$ and $73.47 \%$, respectively. A. castellanii ATCCC 50373 and A2-encoded FLA-CFS (A2-CFS) inhibited the $S 1$ strain $65.64 \%$ and $15.14 \%$, respectively. Against S. aureus ATCC 29213, only A1-CFS showed inhibitory effect (44\%). It was found that $A$. castellanii ATCC 50373-CFS and 
A2-CFS inhibited the S2 strain $26.20 \%$ and $9.24 \%$ respectively. Although A2-CFS inhibited S3 strain (33.33\%), other FLA-CFSs did not show antibacterial effect against this strain. None of the tested FLA-CFSs were found to have an inhibitory effect on $S$. epidermidis ATCC 12228 strain. A3-CFS was found to have no inhibitory effect on any Staphylococcus strain tested. However, it was determined that Staphylococcus strains tested were inhibited by at least one native FLA-CFS (A1-CFS or A2-CFS), except for S. epidermidis ATCC 12228 strain (Figure 3).

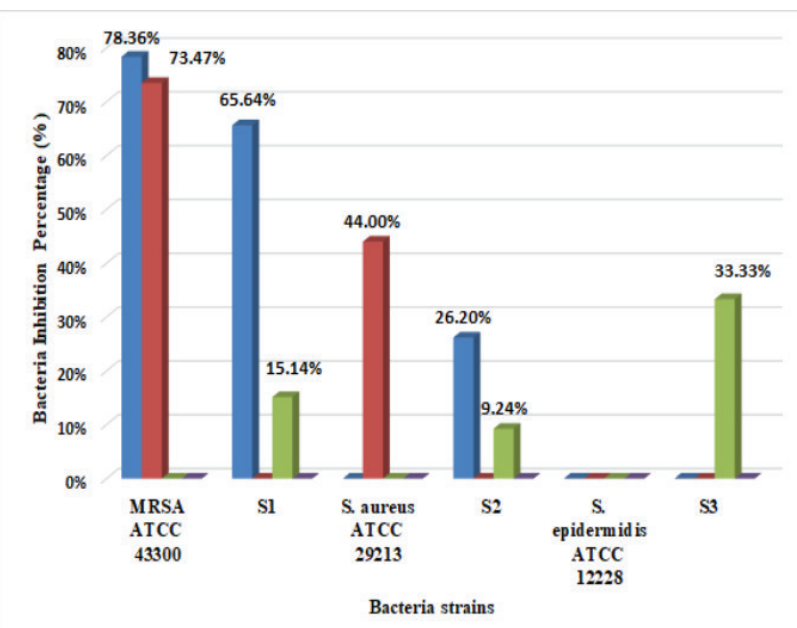

A. castellanii ATCCC $50373=$ A1 $=$ A2 $\approx$ A3

Figure 3. Inhibitory effects of cell-free supernatants (CFSs) against Staphylococcus strains.

According to these data, it was understood that FLA-CFS had more inhibitory effect against $S$. aureus than S. epidermidis generally. S. epidermidis ATCC 12228 strain was not found to be inhibited by any FLA-CFS.

\section{DISCUSSION}

FLA have been attracting the attention of scientists for many years due to their role of regulating microorganism populations while living together with bacteria in the same environment. In the literature, FLA-bacteria relationship has frequently centered on the abilities of amoeba phagocyting bacteria, proliferation of bacteria in amoeba and therefore, causing lysis of them (16$19,39,40)$. However, there are few studies with narrow scope about amoeba inhibiting the proliferation of bacteria by secreting antibacterial substances (apart from phagocytosis) (21-24, 41,42 ). Increasing the number of studies on this topic would help researchers to understand the FLA-bacteria relationship and would enhance microbial ecologic studies, leading to the discovery of new antibacterial substances for bacteria (especially antibiotic-resistant bacteria), which has been a great burden in these times. It was determined that the FLA tested in our study had an inhibitory effect on S. aureus and S. epidermidis (except phagocytosis). The highest inhibition against Staphylococcus bacteria (65.64\%, 78.36\%) was detected with $A$. castellanii ATCC 50373-CFS. A native aquatic FLA strain obtained from our waters (A1-CFS) also yielded a similar (73.47\%, 44\%) inhibition effect. On the contrary, with the other native samples (for A2-CFS) these inhibitions were either low (15.14\%, 9.24\%, $33.33 \%$ ) or (for A3-CFS) non-existent not at all (Figure 3). Nevertheless, these data show us that native FLA have the antibacterial potential against Staphylococcus bacteria. This study is the first one about the interaction/inhibition of FLA-Staphylococcus in which native strains were used. Our studies are ongoing, aiming to investigate more samples to reveal more bacteriostatic or bactericidal effects of FLA isolates against pathogen/potantial pathogen and antibiotic-resistant Staphylococcus. If this kind of effect were found, a new, native, and effective antibacterial substance, which would be applied to inhibition of Staphylococcus that are causing infections in humans, would be discovered.

Similar to our study, Nakışah and Chandrika reported that clinical and environmental (two sample) Acanthamoeba (FLA) lysates showed antibacterial activity against pathogen two S. aureus strains (21). lqbal and co-workers reported that $A$. castellanii-CFS (one sample) was effective against clinical MRSA at $100 \%$ whereas it had a bactericidal activity against vancomycin-resistant $E$. faecalis at $8 \%$. However, it did not show a bactericidal effect against Acinetobacter $s p$., Pseudomonas aeruginosa (22). Souza et al. reported that the relationship between a single clinical MRSA isolate and a single Acanthamoeba polyphaga ATCC 30461 (FLA) strain yielded that FLA culture lysate supported the growth of MRSA, but the same FLA culture supernatant inhibited the growth of the same bacteria (24). Martin et al. investigated the effects of different genus, kind, and origin of FLA-CFS against Staphylococcus aureus (MRSA typed as USA300) and found that Mycobacterium bovis biofilms and A. polyphaga CCAP 1501/18-CFS sample inhibited Staphylococcus aureus to a significant degree (42). As can be seen, there is a very low number of current studies testing a scarce number of strains. However, many more strains (four FLA strains and six Staphylococcus strains) were used in our study, thus making it more comprehensive. Moreover, our study used strains different from the standard strains used in other studies, including native strains. For these reasons, we are of the opinion that the inhibitory effect of FLA CFSs on Staphylococcus bacteria may vary depending on both FLA strains and Staphylococcus strains. The following data obtained from our study (Figure 3) also support this view: i) The tested FLA-CFSs had a greater inhibitory effect against $S$. aureus than S. epidermidis. ii) No FLA-CFS had antibacterial activity against $S$. epidermidis ATCC 12228 strain. iii) A3-CFS had no inhibitory effect on any of the Staphylococcus strains tested. iv) Staphylococcus strains tested were found to be inhibited by at least one native FLA-CFS (A1CFS or A2-CFS), except for the S. epidermidis ATCC 12228 strain). Nevertheless, new studies are needed in which many more Staphylococcus and FLA strains would be used. This would be the only way to shed further light on the subject.

As stated above, since it is thought that the characteristics/abilities (pathogenicity factors) possessed by these microorganisms may play an important role among the factors affecting the relationship between FLA and bacteria living in the same environ- 
ment, the subject should be investigated in detail. For example, it is known that the S. epidermidis ATCC 12228 strain, which was tested in our study and is avirulent, has molecules called bacteriocin that it releases into the external environment (43). This bacterium thus inhibits some species (eg S. aureus) that live in the same environment and are close to it. In our study, the reason why S. epidermidis ATCC 12228 strain was not inhibited by any FLA-CFS is perhaps because of these and similar molecules owned by the bacterium. In addition, it was found that the clinical (virulent) S. epidermidis strain (S3) tested in our study was inhibited by A2-CFS to a low extent (33.33\%). These data suggest that virulent and avirulent $S$. epidermidis strains may be affected in different ways by FLA-CFS. In order to better understand the inhibition effect of FLA-CFS against $S$. epidermidis, new studies are planned in which more strains would be used (both virulent and avirulent S. epidermidis).

Souza Gonçalves et al. found that $A$. castellanii secreted different extracellular vesicles under different stress conditions and the CFS of these vesicles contained 69 proteins (41). In the light of this information, it is thought that the amoeba strains used in our study secrete inhibitory molecules against Staphylococcus strains, and new studies are planned to find the morphological and biochemical characterization of these possible molecules. Subsequent studies should relate to the purification of FLACFS with such activity and the active compound (s) that helped in the discovery of new anti-Staphylococcus active compound (s).

\section{CONCLUSION}

This is the first study showing the anti-Staphylococcus potential of FLA-CFS isolated from Turkey. In order to discover bactericidal FLAs against pathogen/potential pathogen and antibiotic resistant Staphylococcus bacteria, new studies using more strains should be planned.

Peer-review: Externally peer-reviewed.

Conflict of Interest: The authors declare that they have no conflicts of interest to disclose.

Financial Disclosure: This study was supported by Istanbul University Scientific Research Projects Unit with no FBA-201931324.

Author Contributions: Conception/Design of study: Z.Z.; Data Acquisition: S.M.O.; Data Analysis/Interpretation: Z.Z., S.M.O.; Drafting Manuscript: Z.Z., S.M.O.; Critical Revision of Manuscript: Z.Z.; Final Approval and Accountability: Z.Z., S.M.O.

\section{REFERENCES}

1. Thomas V, Herrera-Rimann K, Blanc DS, Greub G, Biodiversity of amoebae and amoeba-resisting bacteria in a hospital water network. Appl Environ Microbiol 2006; 72: 2428-38.

2. Zeybek Z, Üstüntürk M, Binay AR. Morphological characteristics and growth abilities of free living amoeba isolated from domestic tap water samples in Istanbul. Eur J Biol 2010; 69: 17-23.
3. Penz T, Horn M, Schmitz-Esser S. The genome of the amoeba symbiont 'Candidatus Amoebophilus asiaticus' reveals common mechanisms for host cell interaction among amoeba-associated bacteria. J Bacteriol 2010; 1: 541-45.

4. Burak DM. and Zeybek Z. Investigation of legionella pneumophila and free living amoebas in the domestic hot water systems in Istanbul. Turkish J Biol 2011; 35: 679-85.

5. Trabelsi H, Dendana F, Sellami A, Sellami H, Cheikhrouhou F, Neji S, Ayadi A. Pathogenic free-living amoebae: epidemiology and clinical review. Pathol Biol 2012; 60: 399-405.

6. Üstüntürk M. and Zeybek Z. Microbial contamination of contact lens storage cases and domestic tap water of contact lens wearers. Wien Klin Wochenschr 2012; 24: 17-22.

7. Zeybek Z, Dogruoz Gungor N, Turetgen I. Investigation of heterotrophic bacteria, legionella and free - living amoeba in cooling tower samples by fish and culture methods. Eur J Biol 2017; 76: 7-13.

8. Zeybek $Z$ and Türkmen A. Investigation of the incidence of legionella and free-living amoebae in swimming pool waters and biofilm specimens in istanbul by different methods. Microbiol Bull 2020; 54: 50-65.

9. Saburi E, Rajaii T, Behdari A, Kohansal MH, Vazini H. Free-living amoebae in the water resources of Iran: a systematic review. J Parasit Dis 2017; 41: 919-28.

10. Balczun C. and Scheid PL. Free-living amoebae as hosts for and vectors of intracellular microorganisms with public health significance. Viruses 2017; 9: 65.

11. Schuster FL. Cultivation of pathogenic and opportunistic free-living amebas. Clin Microbiol Rev 2002; 15: 342-54.

12. Lambrecht E, Baré J, Van Damme I, Bert W, Sabbe K. Houf K. Behavior of Yersinia enterocolitica in the presence of the bacterivorous Acanthamoeba castellanii. Appl Environ Microbiol 2013; 79: 6407-13.

13. Villanueva MP, Medina G, Fernández H. Arcobacter butzleri survives within trophozoite of Acanthamoeba castellanii. Rev Argent Microbiol 2016; 48: 105-9.

14. Şenler NG, Yıldız İ. Tatı ı Su Protozoonları ve Önemi. Turk J Sci Rev 2010; 3: 7-16.

15. Cateau E, Delafont V, Hechard Y, Rodier MH. Free-living amoebae: What part do they play in healthcare-associated infections?. J Hosp Infect 2014; 87: 131-40.

16. Winiecka-Krusnell J. and Linder E. Bacterial infections of free-living amoebae. Res Microbiol 2001; 152: 613-9.

17. Akya A, Pointon A, Thomas C. Mechanism involved in phagocytosis and killing of Listeria monocytogenes by Acanthamoeba polyphaga. Parasitol Res 2009; 105: 1375-83.

18. Chekabab SM, Daigle F, Charette SJ, Dozois CM, Harel J. Survival of enterohemorrhagic Escherichia coli in the presence of Acanthamoeba castellanii and its dependence on Pho regulon. Microbiologyopen 2012; 1: 427-37.

19. Marciano-Cabral F and Cabral G. Acanthamoeba spp. as agents of disease in humans. Clin Microbiol Rev 2003; 16: 273-307.

20. Greub G, La Scola B, Raoult D. Parachlamydia acanthamoeba is endosymbiotic or lytic for Acanthamoeba polyphaga depending on the incubation temperature. Ann NY Acad Sci 2003; 990: 628-34.

21. Nakisah MA and Chandrika K. Antimicrobial activities of aqueous lysate of Acanthamoeba SPP against selected pathogenic bacteria. Malaysian Appl Bio 2012; 41: 45-9.

22. Iqbal J, Siddiqui R, Khan NA. Acanthamoeba and bacteria produce antimicrobials to target their counterpart. Parasites Vectors 2014; 7: 1-6.

23. Tashmukhambetov B. An investigation of the effects of an antimicrobial peptide on the survival of Acanthamoeba and intracellular bacteria associated with Cystic Fibrosis. University of Essex, Phd Thesis. 2016. 
24. De Souza TK, Soares SS, Benitez LB, Rott MB. Interaction between Methicillin-Resistant Staphylococcus aureus (MRSA) and Acanthamoeba polyphaga. Curr Microbiol 2017; 74: 541-9.

25. CDC. Antibiotic resistance threats in the United States. Cent. Dis. Control Prev. 2019, https://www.cdc.gov/drugresistance/pdf/ threats-report/2019-ar-threats-report-508.pdf

26. Ali SM, Siddiqui R., Ong SK, Shah MR, Anwar A, Heard PJ, Khan NA. Identification and characterization of antibacterial compound(s) of cockroaches (Periplaneta americana). Appl Microbiol Biotechnol 2017; 101: 253-86.

27. Ali $S$, Siddiqui $R$, Khan NA. Antimicrobial discovery from natural and unusual sources. J Pharm Pharmacol 2018; 70: 1287-300.

28. Akbar N, Siddiqui R, Iqbal M, Khan NA. Antibacterial activities of selected pure compounds isolated from gut bacteria of animals living in polluted environments. Antibiotics 2020; 9: 190.

29. Ali SM, Siddiqui R, Sagathevan KA, Khan, NA. Antibacterial activity of selected invertebrate species. Folia Microbiol 2021; 66: 285-91.

30. Borin S, Feldman I, Ken-Dror S, Briscoe D. Rapid diagnosis of Acanthamoeba keratitis using non-nutrient agar with a lawn of $E$. coli. J Ophth Inflame Inf 2013; 3: 1-2.

31. Özpınar N, Özçelik S, Yünlü Ö. Isolation and morphotyping of Acanthamoeba spp. and Vermamoeba spp. from hospital air-conditioning systems. Cumhur Medical J 2017; 39: 369-73.

32. Jacquier N, Aeby S, Lienard J, Greub. Discovery of new intracellular pathogens by amoebal coculture and amoebal enrichment approaches. JoVE 2013; 80 .

33. Schuppler M. How the interaction of Listeria monocytogenes and Acanthamoeba spp. affects growth and distribution of the food borne pathogen. Appl Microbiol Biotechnol 2014; 98: 2907-16.

34. H. P. Agency. Isolation and Identification of Acanthamoeba Species. Nat Stand Method 2014; 17: 1-12.
35. Eroğlu F, Evyapan G, Koltaş is. The cultivation of Acanthamoeba using with different axenic and monoxenic media. Mid Black Sea J Heal Sci 2015; 1: 13-7.

36. Üstüntürk $M$ and Zeybek Z. Amoebicidal efficacy of a novel multi-purpose disinfecting solution: First findings. Exp Parasitol 2014; 145: 93-7.

37. Zeibig E. Clinical Parasitology-E-Book: A Prac App 2012.

38. Huang FC, Shih MH, Chang KF, Huang JM, Shin JW, Lin WC. Characterizing clinical isolates of Acanthamoeba castellanii with high resistance to polyhexamethylene biguanide in Taiwan. J Microbiol Immunol 2017; 50: 570-77.

39. Barker J, Humphrey TJ, Brown MWR. Survival of Escherichia coli 0157 in a soil protozoan: Implications for disease. FEMS Microbiol Lett 1999; 173: 291-5.

40. Garcia, A, Goñi P, Cieloszyk J, Fernandez MT, Calvo-Beguería L, Rubio $E$, Clavel A. Identification of free-living amoebae and amoeba-associated bacteria from reservoirs and water treatment plants by molecular techniques. Environ Sci Technol 2013; 47: 3132-40.

41. Gonçalves DDS, Ferreira MDS, Liedke SC, Gomes KX, de Oliveira GA, Leão PEL, Guimaraes AJ. Extracellular vesicles and vesicle-free secretome of the protozoa acanthamoeba castellanii under homeostasis and nutritional stress and their damaging potential to host cells. Virulence 2018; 9: 818-36.

42. Martin KH, Borlee Gl, Wheat, Jackson WHM, Borlee BR. Busting biofilms: Free-living amoebae disrupt preformed methicillin-resistant Staphylococcus aureus (MRSA) and Mycobacterium bovis biofilms. Microbiology 2020; 166: 695.

43. Jang, IT, Yang M, Kim HJ, Park JK. Novel Cytoplasmic Bacteriocin Compounds Derived from Staphylococcus epidermidis Selectively Kill Staphylococcus aureus, Including Methicillin-Resistant Staphylococcus aureus (MRSA). Pathogen 2020; 9: 87. 